\title{
Radiotherapy Alone is Associated with Improved Outcomes Over Surgery in the Management of Solitary Plasmacytoma
}

\author{
Qi-Wen Li ${ }^{1}$, Shao-Qing Niü, Han-Yu Wang ${ }^{1}$, Ge Wen ${ }^{2}$, Yi-Yang Li $^{1}$, Yun-Fei \\ Xia $^{1}$, Yu-Jing Zhang ${ }^{1 *}$
}

\begin{abstract}
Background: A moderate dose of radiation is the recommended treatment for solitary plasmacytoma (SP), but there is controversy over the role of surgery. Our study aimed at comparing different treatment modalities in the management of SP. Materials and Methods: Data from 38 consecutive patients with solitary plasmacytoma, including 16 with bone plasmacytoma and 22 with extramedullary plasmacytoma, were retrospectively reviewed. 15 patients received radiotherapy alone; 11 received surgery alone, and 12 received both. The median radiation dose was 50Gy. All operations were performed as radical resections. Local progression-free survival (LPFS), multiple myeloma-free survival (MMFS), progression-free survival (PFS) and overall survival (OS) were calculated and outcomes of different therapies were compared. Results: The median follow-up time was 55 months. 5-year LPFS, MMFS, PFS and OS were 87.0\%, 80.9\%, $69.8 \%$ and $87.4 \%$, respectively. Univariate analysis revealed, compared with surgery alone, radiotherapy alone was associated with significantly higher 5 -year LPFS (100\% vs 69.3\%, $p=0.016)$, MMFS (100\% vs 51.4\%, $p=0.006)$, PFS $(100 \%$ vs $33.7 \%, p=0.0004)$ and OS $(100 \%$ vs $70 \%, p=0.041)$. Conclusions: Radiotherapy alone can be considered as a more effective treatment for SP over surgery. Whether a combination of radiotherapy and surgery improves outcomes requires further study.
\end{abstract}

Keywords: Solitary plasmacytoma - radiotherapy - prognostic factors - multiple myeloma

Asian Pac J Cancer Prev, 16 (9), 3741-3745

\section{Introduction}

Solitary plasmacytoma (SP), accounting for $5 \%$ to $10 \%$ of all plasma cell neoplasms (Dimopoulos et al., 2002; Ozsahin et al., 2006), is a rare malignancy defined as a proliferation of monoclonal plasma cells without any systematic involvement. Two subsets are categorized in terms of the location--solitary plasmacytoma of bone (SBP) and extramedullary plasmacytoma (EMP). SBP usually locates in the axial skeleton while EMP occurs in soft tissues, mainly in the head and neck area (Dimopoulos et al., 2002). SP responds well to local treatment. 10-year local control rate reaches to 81-95\% (Chak et al., 1987; Tsang et al., 2001; Reed et al., 2011; Sasaki et al., 2012; Suh et al., 2012). Median survival time is reported to be 7-12 years (Knowling et al., 1983; Chak et al., 1987; Frassica et al., 1989; Liebross et al., 1998; Bachar et al., 2008). However, in spite of a curative treatment, some cases progress to multiple myeloma (MM) eventually, approximately 60\% in SBP (Bataille et al., 1981; Ozsahin et al., 2006; Reed et al., 2011) and 8-64\% in EMP (Strojan et al., 2002; Ozsahin et al., 2006; Bachar et al., 2008; Reed et al., 2011; Suh et al., 2012). Once the disease becomes systemic, the prognosis is similar to patients initially diagnosed with MM, and 5-year OS decreases to $33 \%$ (Mendenhall et al., 1980). Therefore, it is of great importance to distinguish patients on risk of recurrence, and define effective primary therapy to prevent local relapse and MM development.

To our knowledge, radiotherapy (RT) with curative intent is widely accepted, yielding a satisfactory local control rate. Both guidelines from the National Comprehensive Cancer Network (NCCN, version4. 2014) and British Society for Haematology placed RT as a priority for SP (Soutar et al., 2004). Nevertheless, whether surgery plays a beneficial role is still controversial although there is a consensus on surgical intervention in the management of pathological fracture, release of neurological symptoms and surgical biopsy in SBP (Sundaresan et al., 1994; Soutar et al., 2004). Some researchers demonstrated that the addition of surgery contributes to enhancing local control and disease-free survival (DFS) as well (Ozsahin et al., 2006; Kilciksiz et al., 2008). However, in EMP, the effect of surgery is disputed. NCCN guideline recommends RT alone, surgery alone or combination of RT and surgery, while 
Qi-Wen Li et al

the British Society for Haematology guideline considers surgery of the head and neck area inappropriate since the complexity of anatomy. Up to now, whether surgery can benefit patients with SP remains unclear. Our study aimed at dentifying the different therapies with various clinical outcomes.

\section{Materials and Methods}

\section{Subjects}

From 1996 to 2013, data of 38 consecutive patients diagnosed as solitary plasmacytoma and treated in Sun Yat-Sen University Cancer Center were retrospectively analyzed. All patients were under complete examination and met the following criteria: $(i)$ a solitary biopsyconfirmed lesion of neoplastic plasma cells; (ii) no clinical or radiographic evidence of any extra site of disease; evaluated by either a complete skeletal survey or bone scan were reviewed for the evaluation; (iii) bone marrow biopsy showed less than $10 \%$ plasma cells; (iv) absence of hypercalcemia, significant cytopenia, renal dysfunction, elevated immunoglobulin levels, abnormal serum protein electrophoresis, or urine protein electrophoresis . After treatment, patients were followed up and regularly revaluated by clinical assessment and imaging.

The study was performed in compliance with Declaration of Helsinki and was approved by ethics committee.

\section{Statistical analysis}

Endpoints of interest were local progression-free survival (LPFS), multiple myeloma-free survival (MMFS), progression-free survival (PFS) and overall survival (OS). Time was calculated from the date of pathologic diagnosis to the event of interest, which was local progression for LPFS, development to MM for MMFS, death or first disease progression for PFS, and death (from any cause) for OS. The Kaplan-Meier method was used to estimate the survival and recurrence curves. For each end point, detection of statistical differences between selected factors was achieved by the log-rank test. Statistics was conducted by SPSS 18.0. $p$ value $<0.05$ was considered statistically significant.

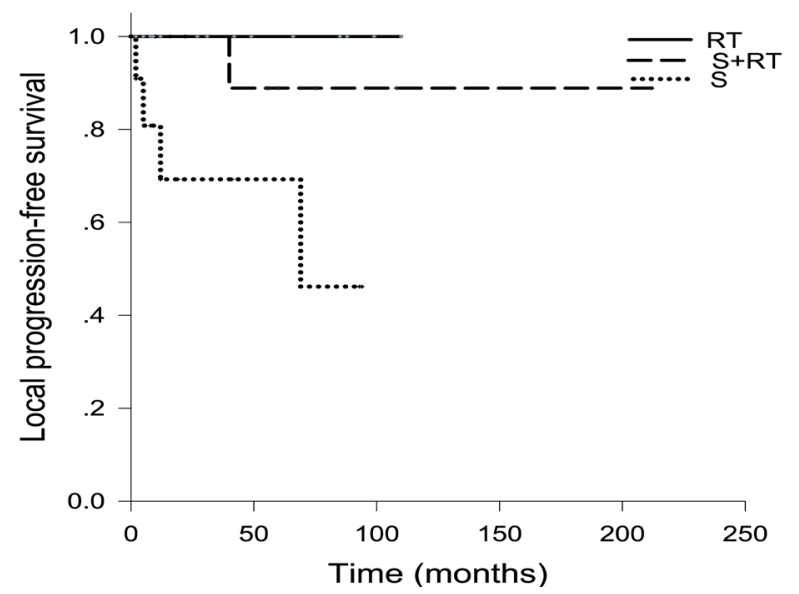

Figure 1. Local Progression-free Survival in 38 Patients with Different Treatment Modalities $(p=0.014)$. RT: radiotherapy, S: surgery

\section{Results}

\section{Patient characteristics}

Characteristics of patients are detailed in Table 1. The median age at diagnosis was 48,42 in SBP and 53 in EMP. 26 Patients were male, and 12 were female. The number of cases of SBP and EMP was 16 and 22, respectively. The most common presentation of SBP was found in the axial skeleton (10/16), while EMP occurred most frequently in the head and neck area (17/22). 9 patients were diagnosed with tumor $>5 \mathrm{~cm} .15$ patients in the study received definitive RT alone. 12 received surgery followed by definitive RT. 11 received surgery alone. Operations were all performed as radical resections. The median radiation dose was $50 \mathrm{~Gy}$.

\section{Local control}

The median follow-up time was 55 (range: 2-212) months. Complete remission was achieved in all patients after primary treatments. 5 patients had locally progressive disease. 5-year LPFS was $87.0 \%$. The median time from diagnosis to local progression was 12 (range:2-59) months. Among these patients, 4 had single local relapse, and 1 patient finally developed MM. According to the univariate analyses, surgery (S) alone is an unfavorable factor of local control compared with RT alone (5-year LPFS: RT vs $\mathrm{S}+\mathrm{RT}$ vs $\mathrm{S}: 100 \%$ vs $88.9 \%$ vs $69.3 \%, p=0.014$, Figure 1. RT vs S: $p=0.016$; RT vs S+RT: $p=0.35$; S+RT vs S: $p=0.063)$. In 16 patients with SBP, 5-year LPFS of RT $v s$ $\mathrm{S}+\mathrm{RT}$ vs $\mathrm{S}$ was $100 \%$ vs $88.9 \%$ vs $100 \%$ ( $p=0.76)$. In 22 patients with EMP, 5-year LPFS was $100 \%$ vs $100 \%$ vs $61.0 \%$ ( $p=0.029$, RT $v s$ S: $p=0.012$; RT vs S+RT: $p=1$; $\mathrm{S}+\mathrm{RT} v s \mathrm{~S}: p=0.4)$.

Table 1. Patient Characteristics

\begin{tabular}{|c|c|c|c|c|c|c|}
\hline \multirow[t]{2}{*}{ Characteristics } & \multicolumn{2}{|c|}{ Total. } & \multicolumn{2}{|c|}{ SBP } & \multicolumn{2}{|c|}{ EMP } \\
\hline & No. & $\%$ & No. & $\%$ & No. & $\%$ \\
\hline \multicolumn{7}{|l|}{ Age } \\
\hline$\geq 50$ & 18 & 47.4 & 6 & 37.5 & 12 & 54.5 \\
\hline$<50$ & 20 & 52.6 & 10 & 62.5 & 10 & 45.5 \\
\hline \multicolumn{7}{|l|}{ Sex } \\
\hline Male & 26 & 68.4 & 12 & 75 & 14 & 63.6 \\
\hline Female & 12 & 31.6 & 4 & 25 & 8 & 36.4 \\
\hline \multicolumn{7}{|c|}{ Subtype and location } \\
\hline SBP & 16 & 42.1 & & & & \\
\hline Axial bone & 10 & 62.5 & & & & \\
\hline Others & 6 & 37.5 & & & & \\
\hline EMP & 22 & 57.9 & & & & \\
\hline Head and neck & 17 & 77.3 & & & & \\
\hline Others & 5 & 22.7 & & & & \\
\hline \multicolumn{7}{|l|}{ Tumor size } \\
\hline$\geq 5 \mathrm{~cm}$ & 9 & 23.7 & 8 & 50 & 1 & 4.5 \\
\hline$<5 \mathrm{~cm}$ & 29 & 76.3 & 8 & 50 & 21 & 95.5 \\
\hline \multicolumn{7}{|c|}{ Radiotherapy/surgery } \\
\hline RT alone & 15 & 39.5 & 4 & 25 & 11 & 50 \\
\hline surgery alone & 11 & 28.9 & 2 & 12.5 & 9 & 40.9 \\
\hline surgery+ RT & 12 & 31.6 & 10 & 62.5 & 2 & 9.1 \\
\hline \multicolumn{7}{|l|}{ Radiation dose } \\
\hline$\geq 45 \mathrm{~Gy}$ & 18 & 66.7 & 8 & 57.1 & 10 & 76.9 \\
\hline$<45 \mathrm{~Gy}$ & 9 & 33.3 & 6 & 42.9 & 3 & 23.1 \\
\hline
\end{tabular}

SBP: solitary plasmacytoma of bone, EMP: extramedullary plasmacytoma, RT: radiotherapy 
Table 2. Univariate Analyses of Prognostic Factors

\begin{tabular}{llccccccccc}
\hline Variable & & $\mathrm{n}$ & 5-y OS $(\%)$ & $p$ & 5-y PFS (\%) & $p$ & 5-y LPFS (\%) & $p$ & 5-y MMFS (\%) & $p$ \\
\hline All patients & & 38 & 87.4 & & 69.8 & & 87 & & 80.9 & \\
Age & $\geq 50$ & 18 & 81.7 & 0.25 & 56.3 & 0.067 & 86.9 & 0.55 & 65.5 & 0.038 \\
& $<50$ & 20 & 91.7 & & 81.6 & & 87.1 & & 94.7 & \\
Gender & Male & 26 & 85.9 & 0.79 & 64.5 & 0.74 & 85.3 & 0.63 & 76.7 & 0.77 \\
& Female & 12 & 90.0 & & 82.5 & & 91.7 & & 90.0 & \\
Subtype & SBP & 16 & 84.4 & 0.42 & 64.5 & 0.65 & 92.9 & 0.16 & 70.0 & 0.13 \\
& EMP & 22 & 94.4 & & 79.5 & & 84.2 & & 94.4 & \\
Tumor size & $\geq 5 \mathrm{~cm}$ & 9 & 100 & 0.15 & 77.8 & 0.60 & 87.5 & 0.56 & 88.9 & 0.78 \\
& $<5 \mathrm{~cm}$ & 29 & 80.9 & & 65.3 & & 88.2 & & 74.4 & \\
Treatment & Surgery+RT & 12 & 87.5 & 0.049 & 71.3 & 0.001 & 88.9 & 0.014 & 80.2 & 0.035 \\
& RT alone & 15 & 100 & & 100 & & 100 & & 100 & \\
& Surgery & 11 & 70.0 & & 33.7 & & 69.3 & & 51.4 \\
\hline
\end{tabular}

SBP: solitary plasmacytoma of bone, EMP: extramedullary plasmacytoma, OS: overall survival, PFS: progression-free survival, LPFS: local progression-free survival, MMFS: multiple myeloma -free survival, RT: radiotherapy

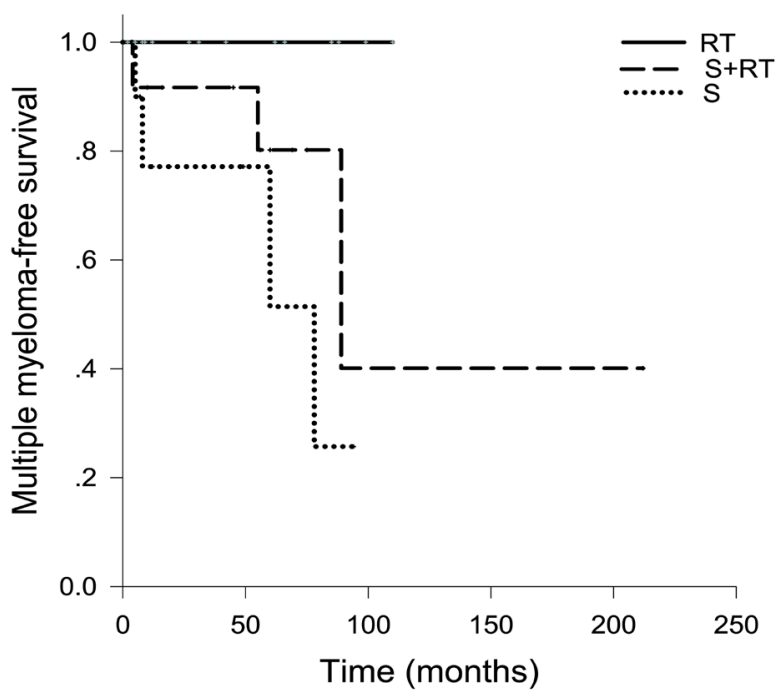

Figure 2. Multiple Myeloma-free Survival in 38 Patients with Different Treatment Modalities $(p=0.035)$. RT: radiotherapy, S: surgery

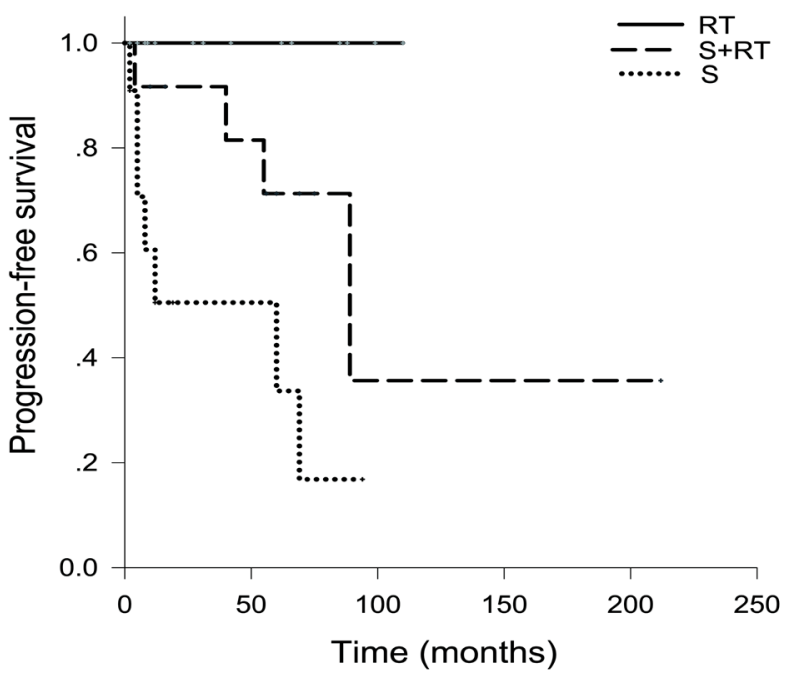

Figure 3. Progression-Free Survival in 38 Patients with Different Treatment Modalities $(p=0.0012)$. RT: radiotherapy, S: surgery

\section{Progression to $M M$}

Progression to MM was observed in 7 patients. The 5-year MMFS was $80.9 \%$. Median time from diagnosis to MM occurrence was 55 (range: 4-89) months. Among

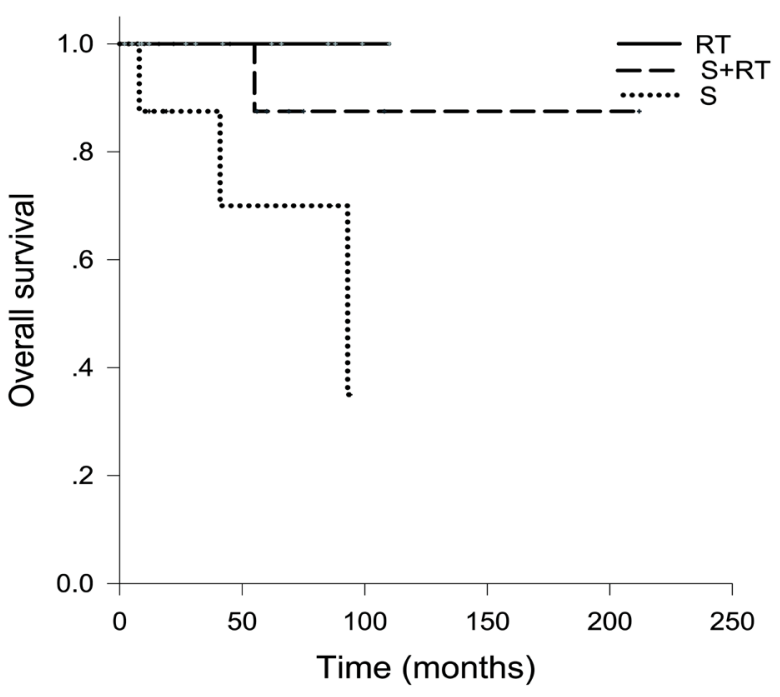

Figure 4. Overall Survival in 38 Patients with Different Treatment Modalities $(p=\mathbf{0 . 0 4 9})$. RT: radiotherapy, S: surgery

these patients, 6 had MM only, and 1 occurred with local progression. 4 patients with MM died of the disease. On univariate analyses, favorable factors for better MMFS were RT (5-year MMFS: RT vs S+RT vs S: $100 \%$ vs $80.2 \%$ vs $51.4 \%, p=0.035$, Figure 2 . RT vs $\mathrm{S}: p=0.006$; RT vs S+RT: $p=0.082$; S+RT vs $\mathrm{S}: p=0.33)$ and age $<50$ (5-year MMFS: $94.7 \%$ vs $65.5 \%, p=0.038$ ). In 16 patients with SBP, 5-year MMFS was $100 \%$ vs $88.9 \%$ vs $100 \%$ $(p=0.053)$. In 22 patients with EMP, 5-year MMFS was $100 \%$ vs $100 \%$ vs $83.3 \%$ ( $p=0.14)$.

Progression-free survival and Overall survival

Overall progression was noted in 11 patients. A total of 4 patients had died. The 5-year PFS and 5-year OS were: $69.8 \%$ and $87.4 \%$. Univariate analyses identified RT as a favorable prognostic factor for 5-year PFS (RT vs S+RT vs S: $100 \%$ vs $71.3 \%$ vs. $33.7 \%, p=0.001$, Figure 3; RT vs S: $p=0.0004$; RT vs S+RT: $p=0.048$; S+RT vs S: $p=0.06)$ and 5-year OS (RT vs S+RT vs S: $100 \%$ vs $87.5 \%$ vs $70.0 \%, p=0.049$, Figure 4; RT vs S: $p=0.041$; RT vs S+RT: $p=0.35$; S+RT $v s$ S: $p=0.13)$. In 16 patients with SBP, 5-year PFS was $100 \%$ vs $70.0 \%$ vs $0 \%(p=0.087)$. 5 -year OS was $100 \%$ vs $87.5 \%$ vs $50.0 \%$ ( $p=0.083)$. In 22 patients with EMP, 5-year PFS was $100 \%$ vs $100 \%$ vs 
Qi-Wen Li et al

50.8\%, ( $p=0.011$, RT vs S: $p=0.005$; RT vs $\mathrm{S}+\mathrm{RT}: p=1$; S+RT vs S: $p=0.31)$. 5-year OS was $100 \%$ vs $100 \%$ vs $83.3 \%(p=0.37)$.

Univariate analyses of prognostic factors is summarized in Table 2.

\section{Discussion}

Solitary plasmacytoma is an uncommon form of plasma cell neoplasms. Due to its rarity, most of our knowledge derives from retrospective studies, which usually regard RT as the most effective therapy. After a moderate (40-50Gy) dose of radiation (Mendenhall et al., 1980; Mendenhall et al., 2003; Kilciksiz et al., 2008), a 5 -year local control rate of $81-95 \%$ could be expected (Ozsahin et al., 2006; Sasaki et al., 2012; Suh et al., 2012). 5-year overall survival rate is reported to be 70$78 \%$ (Frassica et al., 1989; Reed et al., 2011; Suh et al., 2012). 5-year MMFS ranges from 47 to $52 \%$ (Reed et al., 2011; Suh et al., 2012) and 5-year PFS from 43 to 56\% (Frassica et al., 1989; Sasaki et al., 2012; Suh et al., 2012). Similarly, in the current study, though various treatments were used, 5-year LPFS, MMFS, PFS and OS are $87.4 \%$, $69.8 \%, 87.0 \%$ and $80.9 \%$, respectively, comparable to previous studies. When investigating clinicopathological characteristics, age $<50$ was a favorable predictive factor of MM development, which is in agreement with the former researches (Khan et al., 2006; Kocak et al., 2010).

The role of surgery remains controversial in the management of SP. There are only a few reports on surgery alone in SP since the satisfactory local control following RT. Results of surgery alone were not optimal, carrying high rates of local recurrence (78\%) (Ozsahin et al., 2006). Bacher et al. studied 68 patients with head and neck EMP undergoing various treatments (Bachar et al., 2008). To their opinions, although not statistically significant, there was a trend towards increased local control rate in patients treated primarily with radiation than those treated with other modalities, e.g. surgery+RT or chemotherapy. However, according to Strojan et al., no difference on outcomes was found between 15 patients receiving surgery+RT and 12 patients receiving $\mathrm{RT}$ alone. As they considered, close observation is preferred rather than adjuvant RT after R0 resection (Strojan et al., 2002). Interestingly, a research including 57 SBPs and 23 EMPs showed surgery+RT resulted in significantly longer PFS, compared with RT alone (median PFS 7.4 years vs 2.6 years) (Kilciksiz et al., 2008). But this conclusion should be interpreted in caution because EMP, which has a better prognosis intrinsically, is usually associated with a higher portion of excisional biopsy due to its derivation from soft tissue. In our study, RT alone is related to a significantly superior 5-year LPFS, MMFS, PFS and OS compared with surgery alone, supporting RT alone as a primary choice. The delay of definitive RT by the first consideration of surgery may be regarded as an important weakness of surgery+RT strategy.

SBP and EMP represent subgroups of different nature. Additionally, head \& neck and non- head \& neck EMP response to treatment differently. As mentioned before, neither American nor British guideline agrees a routine use of surgery in SBP. But for EMP there is a controversy. Thus, a more detailed analysis is in need. In several studies, patients with head \& neck EMP were free of disease after surgery alone (Kotner et al., 1972; Batsakis et al., 1983). Alexiou et al. reviewed 714 cases $(82.2 \%)$ with lesion found in the upper aerodigestive tract and other 155 $(17.8 \%)$ found in other body regions. Patients with nonupper aerodigestive tumor had similar risk of recurrence no matter they received RT, surgery or combined treatments. But interestingly, for upper aerodigestive tract EMP, higher OS and RFS were detected in those received surgery+RT (Alexiou et al., 1999). More recently, Gerry et al. reported EMP in the head \& neck area responded better to surgery than RT or surgery+RT (Gerry et al., 2013), while some researcher held a contrary opinion (Michalaki et al., 2003; Creach et al., 2009). To be noticed, it is not always possible to remove an EMP completely, especially in the head \& neck area due to the adjacent vital organs structures, highlighting the importance of lesion location in treatment planning. Our study revealed, when SBP and EMP were analyzed independently, the power to detect significant difference was not enough because of the small sample number. But RT still showed its advantage in LPFS and PFS for EMP patients. Since the recommended RT dose for SP is not high, we suggest RT alone as a primary consideration when the toxicity is tolerable. A following resection may be saved for salvage therapy.

Another debate is whether patients on high risk benefit from chemotherapy, surgery+RT or higher RT dose. A previous research demonstrated tumor $\operatorname{size}>5 \mathrm{~cm}$ as a risk factor for local recurrence. The authors believed RT combined with chemotherapy a possible way to improve outcome (Tsang et al., 2001). Some researchers declared local control was increased in head \& neck EMP when dose $>45$ Gy (Tournier-Rangeard et al., 2006). However, Ozsahins et al. (2006) concluded, even for large tumors, higher RT dose failed to make any improvement above $30 \mathrm{~Gy}$. In our study, in 10 cases presented with tumor $>5 \mathrm{~cm}$, only one received $36 \mathrm{~Gy}$ resulted in local failure. Thus, we believe dose escalation is only potentially beneficial, requiring for evidence from well-designed studies. On the other hand, risk factors for MM are: serum $\beta 2$ microglobulin $\geq 3.5 \mathrm{mg} / \mathrm{L}$ (Guo et al., 2013), bone invasion (Ozsahin et al., 2006), involvement of axial bone (Bataille et al., 1981), age (Tournier-Rangeard et al., 2006), monoclonal urinary light chains (Hill et al., 2014) and positive multiparameter flow cytometry (Paiva et al., 2014). Reported by Huang et al., in patients with SP of cervical spine, adjunctive RT following excision obviously decreased MM progression (Huang et al., 2010). Another retrospective study showed favorable effects related to melphalan-based chemotherapy in preventing MM development (Mayr et al., 1990), but others didn't report any benefits of chemotherapy (Holland et al., 1992; Katodritou et al., 2014). Unfortunately, our study doesn't have the potency to explore the effectiveness of intensive therapy in patients of high risk owing to the small number of events.

In conclusion, $\mathrm{RT}$ alone is primarily recommended for SP, while routine use of surgery alone should be avoided. The role of surgery+RT needs to be further studied. 
Although some risk factors have been identified, there is not enough evidence supporting intensive treatment including surgery+RT or high dose RT. Prospective studies with larger sample size are expected to provide higher level of evidence.

\section{References}

Alexiou C, Kau RJ, Dietzfelbinger H, et al (1999). Extramedullary plasmacytoma: tumor occurrence and therapeutic concepts. Cancer, 85, 2305-14.

Bachar G, Goldstein D, Brown D, et al (2008). Solitary extramedullary plasmacytoma of the head and neck-longterm outcome analysis of 68 cases. Head Neck, 30, 1012-9.

Bataille R, Sany J (1981). Solitary myeloma: clinical and prognostic features of a review of 114 cases. Cancer, $\mathbf{4 8 ,}$ 845-51.

Batsakis JG (1983). Pathology consultation. Plasma cell tumors of the head and neck. Ann Otol Rhinol Laryngol, 92, 311-3.

Chak LY, Cox RS, Bostwick DG, Hoppe RT (1987). Solitary plasmacytoma of bone: treatment, progression, and survival. J Clin Oncol, 5, 1811-5.

Creach KM, Foote RL, Neben-Wittich MA, Kyle RA (2009). Radiotherapy for extramedullary plasmacytoma of the head and neck. Int J Radiat Oncol Biol Phys, 73, 789-94.

Dimopoulos MA, Hamilos G (2002). Solitary bone plasmacytoma and extramedullary plasmacytoma. Curr Treat Options Oncol, 3, 255-9.

Frassica DA, Frassica FJ, Schray MF, Sim FH, Kyle RA (1989). Solitary plasmacytoma of bone: Mayo Clinic experience. Int J Radiat Oncol Biol Phys, 16, 43-8.

Guo SQ, Zhang L, Wang YF, et al (2013). Prognostic factors associated with solitary plasmacytoma. Onco Targets Ther, 14, 1659-66.

Gerry D, Lentsch EJ (2013). Epidemiologic evidence of superior outcomes for extramedullary plasmacytoma of the head and neck. Otolaryngol Head Neck Surg, 148, 974-81.

Hill QA, Rawstron AC, de Tute RM, Owen RG (2014). Outcome prediction in plasmacytoma of bone: a risk model utilizing bone marrow flow cytometry and light-chain analysis. Blood, 124, 1296-9.

Holland J, Trenkner DA, Wasserman TH, Fineberg B (1992). Plasmacytoma. Treatment results and conversion to myeloma. Cancer, 69, 1513-7.

Huang WD, Feng DP, Xiao JR, et al (2010). Surgical intervention and radiotherapy outcome of solitary plasmacytoma of cervical spine. Zhonghua Wai Ke Za Zhi, 48, 697-701.

Katodritou E, Terpos E, Symeonidis AS, et al (2014). Clinical features, outcome, and prognostic factors for survival and evolution to multiple myeloma of solitary plasmacytomas: a report of the Greek myeloma study group in 97 patients.

Am J Hematol, 89, 803-8.

Khan MM, Mori M, Sakauchi F, et al (2006). Risk factors for multiple myeloma: evidence from the Japan Collaborative Cohort (JACC) study. Asian Pac J Cancer Prev, 7, 575-81.

Kilciksiz S, Celik OK, Pak Y, et al (2008). Clinical and prognostic features of plasmacytomas: a multicenter study of Turkish Oncology Group-Sarcoma Working Party. Am J Hematol, 83, 702-7.

Knowling MA, Harwood AR, Bergsagel DE (1983). Comparison of extramedullary plasmacytomas with solitary and multiple plasma cell tumors of bone. J Clin Oncol, 1, 255-62.

Kocak E, Ballerini G, Zouhair A, Ozsahin M (2010). Radiation therapy for the solitary plasmacytoma. Turk J Hematol, 27, 57-61.

Kotner LM, Wang CC (1972). Plasmacytoma of the upper air and food passages. Cancer, 30, 414-8.

Liebross RH, Ha CS, Cox JD, et al (1998). Solitary bone plasmacytoma: outcome and prognostic factors following radiotherapy. Int J Radiat Oncol Biol Phys, 41, 1063-7.

Mayr NA, Wen BC, Hussey DH, et al (1990). The role of radiation therapy in the treatment of solitary plasmacytomas. Radiother Oncol, 17, 293-303.

Mendenhall CM, Thar TL, Million RR (1980). Solitary plasmacytoma of bone and soft tissue. Int J Radiat Oncol Biol Phys, 6, 1497-501.

Mendenhall WM, Mendenhall CM, Mendenhall NP (2003). Solitary plasmacytoma of bone and soft tissues. Am J Otolaryngol, 24, 395-9.

Michalaki VJ, Hall J, Henk JM, Nutting CM, Harrington KJ (2003). Definitive radiotherapy for extramedullary plasmacytomas of the head and neck. Br J Radiol, 76, 738-41.

Ozsahin M, Tsang RW, Poortmans P, et al (2006). Outcomes and patterns of failure in solitary plasmacytoma: a multicenter rare cancer network study of 258 patients. Int J Radiat Oncol Biol Phys, 64, 210-7.

Paiva B, Chandia M, Vidriales MB, et al (2014). Multiparameter flow cytometry for staging of solitary bone plasmacytoma: new criteria for risk of progression to myeloma. Blood, 124, 1300-3.

Reed V, Shah J, Medeiros LJ, et al (2011). Solitary plasmacytomas: outcome and prognostic factors after definitive radiation therapy. Cancer, 117, 4468-74.

Sasaki R, Yasuda K, Abe E, et al (2012). Multi-institutional analysis of solitary extramedullary plasmacytoma of the head and neck treated with curative radiotherapy. Int J Radiat Oncol Biol Phys, 82, 626-34.

Soutar R, Lucraft H, Jackson G, et al (2004). Guidelines on the diagnosis and management of solitary plasmacytoma of bone and solitary extramedullary plasmacytoma. $\mathrm{Br} J$ Haematol, 124, 717-26.

Strojan P, Soba E, Lamovec J, Munda A (2002). Extramedullary plasmacytoma: clinical and histopathologic study. Int $J$ Radiat Oncol Biol Phys, 53, 692-701.

Suh YG, Suh CO, Kim JS, et al (2012). Radiotherapy for solitary plasmacytoma of bone and soft tissue: outcomes and prognostic factors. Ann Hematol, 91, 1785-93.

Sundaresan N, Steinberger AA, Moore F, et al (1996). Indications and results of combined anterior-posterior approaches for spine tumor surgery. J Neurosurg, 85, 438-46.

Tournier-Rangeard L, Lapeyre M, Graff-Caillaud P, et al (2006). Radiotherapy for solitary extramedullary plasmacytoma in the head-and-neck region: A dose greater than 45 Gy to the target volume improves the local control. Int J Radiat Oncol Biol Phys, 64, 1013-7.

Tsang RW, Gospodarowicz MK, Pintilie M, et al (2001). Solitary plasmacytoma treated with radiotherapy: impact of tumor size on outcome. Int J Radiat Oncol Biol Phys, 50, 113-20. 